zeit-EKG, Langzeitblutdruckmessung, Lungenfunktionsprüfung und Ultraschalluntersuchungen. Zwei weitere Räume für ambulante Operationen sind noch im Bau.

Von montags bis freitags bieten nun der Unfallchirurg und Chirurg Dr. Richard Mai und die Allgemeinmedizinerin Dr. Susanne Zix regelmäßige Sprechstunden an. Beide Ärzte sind angestellt. Sie werden unterstützt von zwei Medizinischen Fachangestellten, einer davon ist zugleich Röntgenassistent.

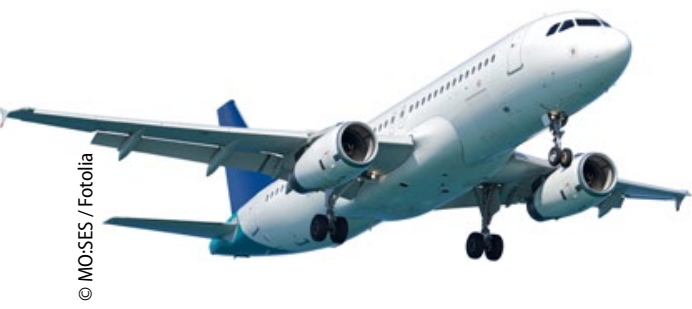

Irgendwann sollen auch Flugreisende eine wichtige Patientengruppe bilden.

Für die 49-jährige Hausärztin ändert sich mit der neuen Tätigkeit nicht viel. Sie war lange im Berliner Haus der Gesundheit angestellt, bevor sie nach Potsdam und jetzt nach Schönefeld wechselte. „Ich schätze es sehr, mit der Klinik zusammenzuarbeiten“, sagt Zix. Ein Vorteil der Arbeit unter dem Dach derselben Organisation sei die schnelle Wei- tervermittlung von Patienten mit komplizierten Krankengeschichten zu den Experten. So konnte sie einen jungen $\mathrm{Pa}$ tienten, der seit zwei Jahren Durchfälle hatte, an die gastroenterologische Sprechstunde des Klinikums verweisen.

\section{Hausärztin in Anstellung}

Doch nicht nur von ihrem neuen Arbeitgeber, auch von ihrem neuen Praxisstandort ist sie überzeugt: „Die Versorgung war hier nicht sehr gut. Ich denke schon, dass wir hier gebraucht werden“, sagt Zix. Die gebürtige Berlinerin übt die Hausarztmedizin gern angestellt aus. „Wenn man Familie hat, ist es vorteilhaft, wenn man sich im Beruf nicht um alles kümmern muss", sagt sie. Jetzt freut sie sich auf die abwechslungsreiche Arbeit am Großflughafen in spe.

Die MVZ-Tochter des Bergmannklinikums will die Entwicklung des Schönefelder MVZ zunächst beobachten. Schönefeld ist der dritte Standort nach Potsdam und Kleinmachnow im Süden von Berlin, wo das Klinikum ein MVZ betreibt. Rund zwei Jahre wird es nach Einschätzung des kaufmännischen Geschäftsführers Thomas Pfeiffer dauern, bis die Flughafen-Praxen so angelaufen sind, dass sie den Break Even erreichen können. Dann ist nicht ausgeschlossen, dass weitere MVZ-Praxen in das Airport Center einziehen.

Angela Mißlbeck

\title{
Bundesregierung sieht MVZ im Aufwind
}

Der verbeamtete Gesundheitsstaatssekretär Lutz Stroppe hat die Medizinischen Versorgungszentren (MVZ) in Deutschland gelobt. Ihre Akzeptanz steige zusehends, betonte er beim Praktikerkongress des Bundesverbands MVZ (BMVZ) in Berlin. Auch in der Gesundheitspolitik der CDU, der er selbst angehört, habe sich ein Sinneswandel vollzogen: „Die Diskussion in der Union hat sich mit Blick auf die MVZ ganz erheblich verändert", sagte Stroppe. Man habe gesehen, dass MVZ neue Möglichkeiten der Versorgung schaffen. Das sei eine sehr erfreuliche Entwicklung.

„Kooperation und Koordination auf unterschiedlichen Ebenen über Fach- und Sektorengrenzen hinweg sind Schlüssel, um die medizinische Versorgung zu verbessern", zeigte sich Stroppe überzeugt. Die Koalition habe mit dem Versorgungsstärkungsgesetz versucht, die Möglichkeiten für MVZ zu erweitern und die Voraussetzungen für eine weitere positive Entwicklung zu schaffen.

Als „wichtige Weiterentwicklung“ bewertete der Staatssekretär die Möglichkeit zur Gründung fachgleicher MVZ. „Ein Schritt, um das Instrument weiter in die Fläche zu bringen“ seien die Möglichkeiten für Kommunen, MVZ zu gründen.

- ami

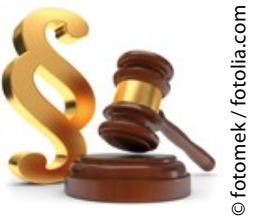

Kasse zahlt nur Fahrten zum nåchsten Arzt

Schwer Gehbehinderte haben keinen Anspruch auf Fahrtkostenerstattung zu einem beliebigen Arzt. Die gesetzlichen Krankenkassen müssen nur das Taxi zur nächstgelegenen Praxis bezahlen, wie jetzt das Bundessozialgericht (BSG) in Kassel entschied. Ausnahmen gebe es nur bei zwingenden, insbesondere medizinischen Gründen. Damit scheiterte ein Gehbehinderter mit seinen Einwänden gegen die von der DAK vorgeschlagenen Praxen: Eine habe keine Hausbesuche angeboten, ein Lungenmediziner spreche einen schwer verständlichen Dialekt, ein Augenarzt könne keine Laser-Behandlungen durchführen. Das BSG sah keine Behinderung der freien Arztwahl, wenn die Kasse das Taxi nur bis zu diesen Ärzten bezahlen wolle. $\quad-$ mwo

- BSG, Az::B 1 KR 27/14R

\section{Patientinnen heimlich} gefilmt: Berufsverbot

Weil er heimlich intime Fotos von $\mathrm{Pa}$ tientinnen mit einer Kugelschreiberkamera gemacht hat, hat das Landgericht Osnabrück einen Hausarzt zu einer Bewährungsstrafe von zwei Jahren verurteilt - und ihm ein dreijähriges Berufsverbot auferlegt. Zudem muss der 62-Jährige 75.000 Euro an gemeinnützige Organisationen zahlen.

Die Kammer sah es als erwiesen an, dass der Allgemeinmediziner in 58 Fällen seine Patientinnen heimlich gefilmt und in 12 Fällen sogar sexuell missbraucht habe. Außerdem wurden auf seinem Computer große Mengen kinderpornografischen Materials gefunden, das er auch über Tauschbörsen anderen zugänglich gemacht hat.

Zugunsten des Arztes wirkte sich sein umfassendes Geständnis ebenso aus wie die Tatsache, dass er bereits über 160.000 Euro an Geschädigte gezahlt hat. - chy

- LG Osnabrück, Az.: 10 KLs 10/15 